with the crystallographic model. The graphics library is independent of the crystallographic library and provides an interface sufficient for the implementation of complex graphical objects.

\section{MS30 O4}

News from DirAx and Indexing Jürgen Kopf Department of Chemistry, University of Hamburg, Germany. E-mail: kopf@xray.chemie.uni-hamburg.de

\section{Keywords: Auto-indexing; Dirax-algorithm; Programming}

Auto-indexing routines [1] have been used extensively for single crystal diffractometers with point-detector devices in the seventies and eighties of the last century. Without indexing routines data collection is impossible on such instruments. They depend on the accurate measurements of the diffractometer angles of a few selected (between 10 to 15$)$ reflections.

In cases of non-merohedral twinning these techniques fail generally to find suitable cell parameters. By exclusion or replacement of unfavorable reflections, changing tolerances or other, mostly undocumented activities, indexing problems can be solved sometimes.

In 1992 Duisenberg [2] described a new algorithm for single-point-detector data. The method presented by Duisenberg takes the whole reflection list and constructs so-called "direct axes" vectors from all data. Then, the projections of the reciprocal lattice points onto these vectors are calculated and each line projection is searched for the one-dimensional lattice with the shortest period that represents the maximum number of projected reflections. The final primitive cell is determined from a reduced set of properly averaged "direct axes" vectors that give the best fit.

Nowadays only single crystal diffractometers with area detectors are in use, but an intrinsic problem of areadetector data is that the measured positions are less accurate than measured with point-detector devices. Therefore, other approaches are necessary for fast and reliable indexing in these cases.

A new method for auto-indexing of area-detector data was introduced by Steller et al. [3] in 1997. Steller also constructs "direct axes" vectors, but unlike the method of Duisenberg his routine tests all possible directions over a hemisphere and analyzes the frequency distribution of these vectors in each case. Also unlike the procedure of Duisenberg the periodicity is determined using fast fourier transform.

The concept of the reciprocal lattice is very important in xray-crystallography. In connection with the development of a new graphically oriented program for the visualization and the 3D presentation of the reciprocal lattice both algorithms were re-implemented. This program, called QTRECLAT, uses the Qt 4 and the OpenGL libraries and can handle an almost unlimited number of reflections. It reads a file that contains all relevant information about the positions and the intensities of the reflections, as can be produced with the Bruker-AXS SMART software. With the help of two projections on the $\mathrm{x}$ - and $\mathrm{y}$-axis the extraction of periodic information of the reciprocal lattice is trivial for the human eye. However, extracting the periodic length for a computer program is much more difficult to realize. The program is written in $\mathrm{C}++$ using the Qt 4 class library [4]. Qt is the leading framework for native crossplatform application development that run on Windows, Unix/Linux and Mac OS X.

An important part of the program QTRECLAT is the refine-ment of the orientation matrix. This allows the definitive discrimination between reflections of the basic lattice and possible twin or other satellite reflections. Another feature of the program is that it can consider the intensity distribution of the reflections. Thus, the program may also serve as a good starting point for the following integration procedures.

[1] Sparks R.A., Crystallographic Computing Techniques, 1976, 452.

[2] Duisenberg A.J.M., J. Appl. Cryst., 1992, 25, 92.

[3] Steller I., Bolotovsky R., Rossmann M.G., J. Appl. Cryst., 1997, 30, 1036.

[4] http://www.trolltech.com/

\section{MS30 O5}

Comparing Entire Crystal Structures: Structural Genetic Fingerprinting Andrew Parkin ${ }^{a}$, Gordon Barr ${ }^{\mathrm{a}}$, Wei Dong ${ }^{\mathrm{a}}$, Christopher J. Gilmore ${ }^{\mathrm{a}}$, Dylan Jayatilaka ${ }^{\mathrm{b}}$, Joshua J. McKinnon ${ }^{\mathrm{b}}$, Mark A. Spackman ${ }^{\mathrm{b}}$ and Chick C. Wilson ${ }^{\mathrm{a}}{ }^{\mathrm{a}}$ WestCHEM Research School, Dept. of Chemistry, University of Glasgow, University Avenue, Glasgow G12 8QQ, UK. ${ }^{\mathrm{b}}$ Chemistry - M313, School of Biomedical, Biomolecular \& Chemical Sciences, University of Western Australia, Crawley, WA 6009, Australia. E-mail: a.parkin@chem.gla.ac.uk

\section{Keywords: Structural Comparison, Structural Similarity, Cambridge Structural Database}

A method is described that is both robust and generally applicable, which allows the calculation of a similarity index between whole molecular crystal structures [1]. It is based on the use of fingerprint plots derived from Hirshfeld surfaces coupled with cluster analysis and associated multivariate statistics. Using this formalism, it is possible to show quantitatively that naphthalene is more similar to anthracene than to benzene, and moreover that benzodicoronene is more similar to anthrabenzonaphthopentacene than naphthalene is to anthracene. Whereas the correlation coefficients themselves obtained say nothing about the ways in which the patterns of intermolecular interactions are similar or different for two different structures, the fingerprint plots do contain such information. For the first time structural analysts have a robust method for quantifying structural similarities of whole molecular crystal structures; this has been termed 'Structural Genetic Fingerprinting'. A number of examples will be presented. There is no reason why this method cannot be applied to datasets consisting of many hundreds or even thousands of structures.

[1] Parkin A., Barr G., Dong W., Gilmore C.J., Jayatilaka D., McKinnon J.J., Spackman M.A., Wilson C.C., CrystEngComm., 2007, Accepted for publication. 